\title{
Correction: What is axial spondyloarthritis? A latent class and transition analysis in the SPACE and DESIR cohorts. A meta- analysis and functional study
}

Sepriano A, Ramiro S, van der Heijde D, et al. What is axial spondyloarthritis? A latent class and transition analysis in the SPACE and DESIR cohorts. A meta-analysis and functional study. Ann Rheum Dis 2020;79:324-31.

The values for some of the features in the SPACE cohort were switched between patients included and excluded in the analysis. The appropriate corrections were made in table 1 and online supplementary table S1. The text in the results section was also corrected: "In SPACE, included patients were less likely to be HLA-B27 positive (37\% vs5 7\%) (...)”.

Table 1 Baseline patient characteristics in the space and DESIR cohorts

\begin{tabular}{lcc}
\hline & $\begin{array}{c}\text { Space } \\
(\mathbf{n}=465)\end{array}$ & $\begin{array}{c}\text { Desir } \\
(\mathbf{n}=576)\end{array}$ \\
\hline Age at baseline (years) & $31(8)$ & $33(8)$ \\
\hline Male gender & $161(35)$ & $1.5(0.8)$ \\
\hline Symptom duration (years) & $1.8(2.3)$ & $358(62)$ \\
\hline ASAS axSpA criteria & $172(37)$ & $269(47)$ \\
\hline axSpA according to Rheumatologist* & $136(30)$ & $320(56)$ \\
\hline ASAS pSpA criteria & $182(39)$ & $443(77)$ \\
\hline ASAS SpA criteria† & $249(54)$ & $153(27)$ \\
\hline Sacroilitis on MRI-SIJ (ASAS) & $64(14)$ & $25(4)$ \\
\hline BME on MRI-spine ( $\geq 5$ lesions) & $21(5)$ & $78(14)$ \\
\hline Radiographic sacroiliitis (mNY) & $38(8)$ & $39(7)$ \\
\hline$\geq 1$ syndesmophyte on X-spine & $15(3)$ & $169(29)$ \\
\hline Elevated CRP ( $\geq$ mg/L) & $118(25)$ & $491(85)$ \\
\hline Good response to NSAIDs ever & $189(41)$ & $122(21)$ \\
\hline Peripheral arthritis ever & $76(16)$ & $78(14)$ \\
\hline Dactylitis ever & $23(5)$ & $261(45)$ \\
\hline Heel enthesitis ever & $91(20)$ & \\
\hline
\end{tabular}

\begin{tabular}{|c|c|c|c|c|}
\hline \multicolumn{5}{|l|}{ Supplementary Table } \\
\hline & All $(n=748)$ & $\begin{array}{l}\text { Included in LCA } \\
(\mathrm{n}=465)\end{array}$ & $\begin{array}{l}\text { NOT included in } \\
\text { LCA }(n=283)\end{array}$ & $P$ value \\
\hline Age at baseline (years) & $31(8)$ & $31(8)$ & $30(8)$ & 0.35 \\
\hline Male gender & $287(39)$ & $161(35)$ & $126(45)$ & 0.01 \\
\hline Symptom duration (years) & $2(2)$ & $2(2)$ & $2(2)$ & 0.93 \\
\hline Inflammatory back pain & $513(69)$ & $308(66)$ & $205(74)$ & 0.027 \\
\hline ASAS axSpA criteria & $308(42)$ & $172(37)$ & $133(52)$ & $<0.01$ \\
\hline ASAS pSpA criteria & $281(39)$ & $182(39)$ & $99(39)$ & 0.93 \\
\hline ASAS SpA criteriat & $412(57)$ & $249(54)$ & $163(64)$ & 0.01 \\
\hline axSpA according to Rheumatologist* & $255(35)$ & $136(30)$ & $119(45)$ & $<0.01$ \\
\hline HLA-B27 & $328(44)$ & $172(37)$ & $156(57)$ & $<0.01$ \\
\hline Dactylitis ever & $41(6)$ & $23(5)$ & $18(6)$ & 0.39 \\
\hline Uveitis ever & $60(8)$ & $33(7)$ & $27(10)$ & 0.21 \\
\hline Family history of SpA & $317(43)$ & $194(42)$ & $123(44)$ & 0.50 \\
\hline Inflammatory bowel disease ever & $51(7)$ & $35(8)$ & $16(6)$ & 0.34 \\
\hline Psoriasis ever & $95(13)$ & $54(12)$ & $41(15)$ & 0.23 \\
\hline Peripheral arthritis ever & $117(16)$ & $76(16)$ & $41(15)$ & 0.56 \\
\hline Heel enthesitis ever & $146(20)$ & $91(20)$ & $55(20)$ & 0.96 \\
\hline Current arthritis / any enthesitis / dactylitis & $475(64)$ & $317(68)$ & $158(58)$ & $<0.01$ \\
\hline Elevated CRP ( $\geq 6 \mathrm{mg} / \mathrm{L}$ ) & $182(25)$ & $118(25)$ & $64(24)$ & 0.57 \\
\hline Good response to NSAIDs ever & $327(45)$ & $189(41)$ & $138(53)$ & $<0.01$ \\
\hline Number of SpA features $(0-9) \ddagger$ & $2(1)$ & $2(1)$ & $2(1)$ & 0.35 \\
\hline Radiographic sacroiliitis (mNY) & $55(9)$ & $38(8)$ & $17(11)$ & 0.26 \\
\hline Sacroiliitis on MRI-SIJ (ASAS) & $106(18)$ & $64(14)$ & $42(30)$ & $<0.01$ \\
\hline BME on MRI-spine ( $\geq 5$ lesions) & $25(4)$ & $21(5)$ & $4(3)$ & 0.42 \\
\hline$\geq 1$ syndesmophyte on $X$-spine & $20(4)$ & $15(3)$ & $5(9)$ & 0.06 \\
\hline
\end{tabular}




\begin{tabular}{lcc}
\hline & $\begin{array}{l}\text { Space } \\
(\mathbf{n}=465)\end{array}$ & $\begin{array}{l}\text { Desir } \\
(\mathbf{n}=576)\end{array}$ \\
\hline HLA-B27 & $172(37)$ & $345(60)$ \\
\hline Family history of SpA & $194(42)$ & $250(43)$ \\
\hline Psoriasis ever & $54(12)$ & $99(17)$ \\
\hline Uveitis ever & $33(7)$ & $52(9)$ \\
\hline Inflammatory Bowel Disease ever & $35(8)$ & $25(4)$ \\
\hline Current arthritis / any enthesitis / dactylitis & $317(68)$ & $398(69)$ \\
\hline Inflammatory back pain & $308(66)$ & $576(100)$ \\
\hline Number of SpA features (0-9)‡ & $2(1)$ & $3(1)$ \\
\hline
\end{tabular}

Values are mean (SD) for continuous variables or number (\%) for binary variables. SpA features are positive if 'ever present' (any time in the past and/or baseline). *Clinical diagnosis of axSpA at baseline with a level of confidence $>7$; Missing data SPACE: axSpA according to Rheumatologist $(n=454)$; Symptom duration $(n=461)$; missing data DESIR: axSpA according to Rheumatologist $(n=576)$.

tfulfilment of either ASAS axSpA or ASAS pSpA classification criteria.

‡peripheral arthritis, heel enthesitis, dactylitis, psoriasis, uveitis, inflammatory bowel disease, good response to NSAIDs, elevated CRP and family history of SpA.

ASAS, Assessment of SpondyloArthritis international Society; axSpA, axial spondyloarthritis; BME, bone marrow oedema; CRP, C-reactive protein; mNY, modified New York

criteria; MRI, magnetic resonance imaging; NSAIDs, nonsteroidal anti-inflammatory drugs; pSpA, peripheral spondyloarthritis; SD, standard deviation; SIJ, sacroiliac joints;

$X$-spine, radiograph of the spine.

(C) Author(s) (or their employer(s)) 2020. No commercial re-use. See rights and permissions. Published by BMJ.

Ann Rheum Dis 2020;79:e78. doi:10.1136/annrheumdis-2019-216516corr1

(A) Check for updates 